\title{
Arthur S M Lim
}

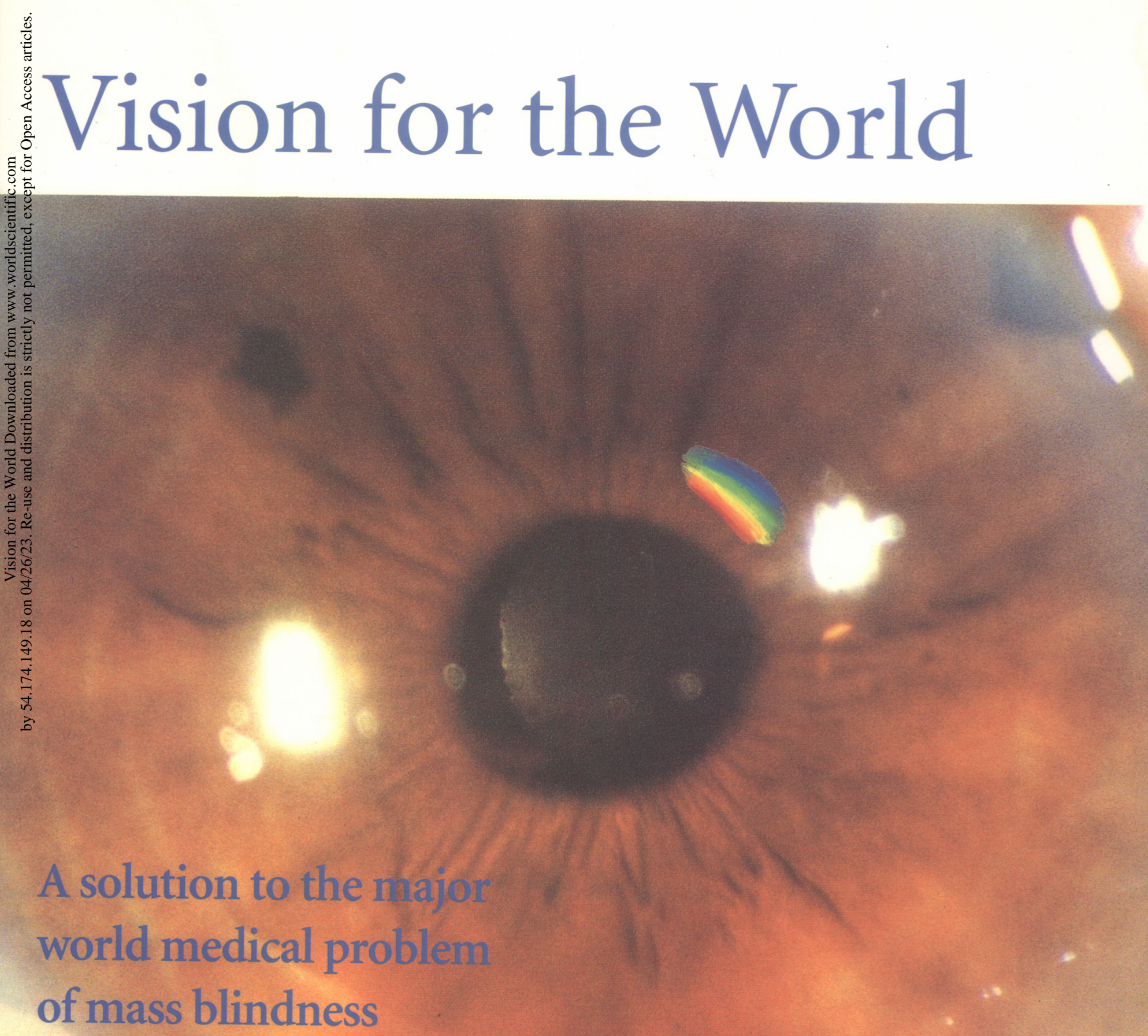


This page is intentionally left blank 
Published in 1996 by

World Scientific Publishing Co. Pte Ltd.

P O Box 128, Farrer Road, Singapore 912805

USA office: Suite 1B, 1060 Main Street, River Edge, NJ 07661

UK office: 57 Shelton Street, Covent Garden, London WC2H $9 H E$

on behalf of the

Singapore National Eye Centre

11 Third Hospital Avenue, Singapore 168751

\section{Vision for the World, 1st Edition}

Copyright (C) 1996 by Singapore National Eye Centre and World Scientific C0. Pte Ltd. All rights reserved. This book, or parts thereof, may not be reproduced in any form or by any means, electronic or mechanical, including photocopying, recording or any information storage and retrieval system now know or be invented, without written Dermission from the Publishers.

Singapore National Eye Centre
World Scientific Co. Pte Ltd.
Book code 2974
Design and Production by Words 9

Printed in Singapore 


\title{
Vision for the World
}

\author{
Eye surgeons' solution to mass blindness \\ - a major world medical problem.
}

\section{Arthur S M Lim}

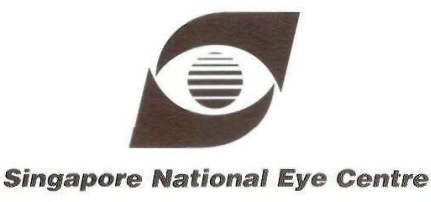

No world Scientific Publishing Co Pte Ltd

Singapore • New Jersey • London • Hong Kong 


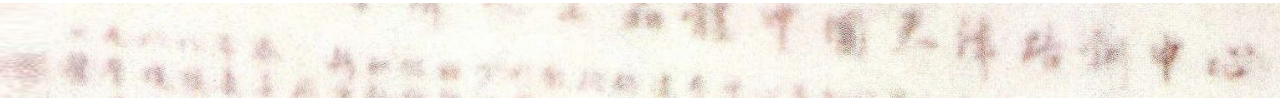

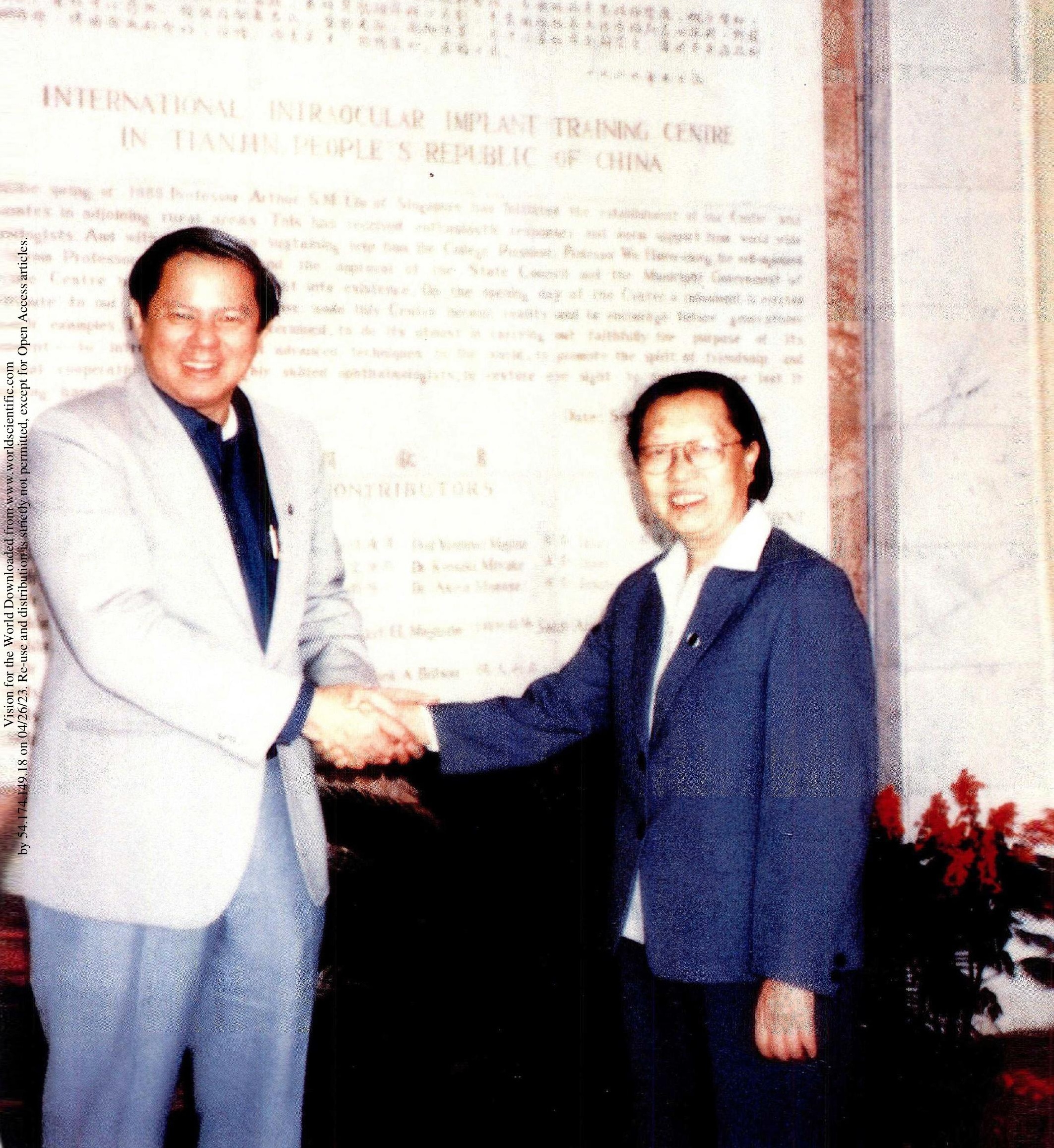




\title{
Dedication
}

"My vision, her effort."

\author{
I dedicate this book to Professor Yuan Jia-Qin who is my foremost partner in my \\ battle of ten years against cataract blindness. Professor Yuan Jia-Qin, a dynamic, \\ dedicated, skilful, determined, highly intelligent, leader of ophthalmology \\ who established a centre which will be a model to solve one of the world's most \\ important medical problems.
}

To all the eye surgeons of the world, I also dedicate my book and hope that it will be of some value in their own missions to make the world a better place.

Abhaykumar Vasavada, Abiose Adenike, Absolon M J, Agarwal Lalit, Akef Al-Maghraby, Albert Daniel M, Allewaert R M, Alpar John, Amalric Pierre, Amar Leo, Anderson R D, Anis Aziz, Apple David, Arentsen Juan, Arnott Eric J, Aron-Rosa Daniele, Avetisov Eduard S, Azar Robert, Baikoff Georges, Barnet R W, Barraquer Joaquin, Barraquer Jose I, Barret Graham, Barron Gerald I, Bechert Ul Charles H, Becker Stanley C, Benjamin Frederick A, Beyzaii Saeed, Bieshevval Kommer, Billson Frank A, Binkhorst Cornelius, Bird Alan C, Blodi Frederick C, Bloomberg Leroy B, Blumenthal Michael, Bochove J Van, Bose J, Boyd Benjamin F, Brancato Rosario, Bruckner Howard L, Buratto Lucio, Byron Herve, Caramazza Roberto, Charleux Jaques, Chen Tetsaw, Chirambo Moses C, Choyce Peter, Christy Norval, Clayman Henry M, Condon Patrick, Constable Ian J, Contreras Francisco, Coop Harold V, Corydon Leif, Coscas Gabriel, Coster Douglas J, Crock Gerard W, Cruickshanks Bryan, Cuendet J F, Cumha-Vaz Jose, Dallas Neil, Dardenne Ulrich, Darin John, Demers Jean Paul, Demong T, Deutman August F, Diallo Joseph, Dodick Jack, Dominguez Alfredo, Donald R, Dong Ho Youn, Dossi Fabio, Douvas N G, Draeger Jorg, Drance Stephen M, Drews Robert, Duffner Lee R, Dulaney David D, Duque-Estrada W, Ellingson F T, Emery Jared, Epstein Edward, Esente Ivan, Fanta Helmut, Faulborn J, Faulkner James, Fechner P U, Fernandez S Tony, Ffytche Timothy J, Fitterman Herbert N, Franks Stan, Freeman Jerte M, Freyler Heinz, Frezzotti Renato, Fronimopoulos John, Frumar Kim D, Fukado X, Fyodorov Svyatoslav, Galand Albert, Galin Miles A, Gallenga Pier E, Galli Lorant, Garcia Charles, Gass Donald, Gilmore I E, Gimbel Howard, Giovinco Joseph, Gloor Balder, Gould Herbert, Grabow Harry B, Gregersen Eilif, Grom Edward, Gyorffy Istvan, Hagan III John C, Haining W M, Hara Tsutomo, Hardenbergh Firmon, Hardia Pratap Singh, Harris Williams, Hartani Dehbia, Hayano S, Hayashi Fumihiko, Henkes Harold E, Hertzog Francis, Hillman Jeffrey, Hirshman Henry, Ho Patrick C P, Hoffer Kenneth, Holmberg Ake Docent, Hoskins H Dunbar, Huber Christophe, Hunkeler fohn D, Husain Rabiul, Ilyas Sidarta, Lzak Milan, Jacobi Carl, Jae Ho Kim, Jaeger Wolfgang, Jaffe Norman, Jamieson Ian Wallace, Jasdliwala Juzar M, Jean-Jacques de Laey, Jones Larry W, Junge J, Kadi lozef, Keates Richard, Kelman Charles, Keshmahinder Singh, Khain Akhtar Jamal, Khan M Daud, Khan Mohd Daud, Khoo Chong Yew, Kirsch Ralph, Knorz Michael C, Ko Liang-Shi, Koch Douglas, Kogure Fumio, Korver C, Kothari Kulin, Kraff Manus, Krasnov M M, Kratz Richard, Kwitko Marvin, Kyung Hwan Shyn, Lcbuisson Alexandra, Lecaillon-Thibon B, Lee Kyoung Hum, Lee Shui-Mei, Lee X C, Leonard Paul, Lemer 
Melvin, Leung William, Li Feng Min, Li Zi-Liang, Liang Yu-Sung, Lichter Paul R, Lieb W A, Light David, Lindstrom Richard, Lowe Ronald F, Lu Dao-Yan, Luntz Maurice H, Madan Mohan, Madan P Upadhyay, Maida Jerry, Majima Yoshinao, Makar M S, Makuloluwa C A B, Malbran Enrique, Manual Lewis C, Marsetio Mardiono, Masuda Kanjiro, Matheen T M, Maumenee Edward A, Maumenee Irene H, Mawas Edouard, McCannel Malcolm, McCulloch Clement, McIntyre D J, Mehta H K, Mehta Keiki R, Menezo Jose-Luis, Merte H J, Mestdagh V der Neste, Mester U, Michaelis Mary K, Mishima Saiichi, Miyake Kensaku, Moizz Abdul, Momose Akira, Morcher K, Morrish Desmond, Moses Lyle, Muer Guy Charles, Mumtaz Raja, Murthy A S R, Murube Del Castillo, Nagata Makoto, Nagpal P N, Nakajima Akira, Nam Ho Baek, Naumann G O C, Naumann Gottfried, Naval Cosme, Newell Frank W, Nikeghbzali A, Nishi Okihiro, Noonan David J, Obstbaum Stephen, Oleksy Stanley P, Osher M S, Oshima Kenji, Otto A J, Paganoni Camillo, Pararajasegaram R, Parelman Alan G, Parker R W, Parliyama Matthiys, Pearce John, Peckar C O, Percival Piers, Perdriel Georges, Perrit Richard, Petkus Raymond, Philipson Bo, Phua Raymond, Pierse Dermot, Pokhrel R P, Pouliquen Yves, Pouliquen Yves J M, Pretorius Marius, Puchkovskaya N A, Quintana Manuel, Radjamin R K Tamin, Rhee Sang-Wook, Ribeiro-DaSilva J, Rice Noel S C, Rich W, Ridgway A E A, Ridley Harold, Ring Calvin, Ring Peter, Rodriguez Alvaro, Romeo B Esperitu, Romeo V Fajardo, Rommel Joseph, Roper-Hall Michael, Rosen Emanuel, Royer Jean, Ryan Stephen J, Sachsenwager Rudolf, Samiee Hossein, Saraf G K, Saraux Henry, Schimmelpfennig B, Schott Klaus, Scuderi Giuseppe, Sellyei Jr Louis, Selm J L van, Selvarajah S, Severin Sanford, Sheets John, Shepard Dennis, Shimizu Kimiya, Shimizu Koichi, Shyn Kyung Hwan, Siam Abdel Latif, Simcoe C Williams, Simpson E Randolph, Singer David, Singh Daljit, Singh Inderjit, Singh Mohinder, Sinskey Robert, Sivagnanam C, Smith S Gregory, Solomon Leon, Sommer Alfred, Soultanakis Paul E, Sourdille Philippe, Spivey Bruce E, Stark Walter, Starr Arthur, Steele A. D Mc, Stein Harold, Stenevi Ulf Docent, Straatsma Bradley R, Stubbs G Maxwell, Stucchi Carlo A, Sun Huimin, Svarc Emile, Tackman Ramon N, Takeuchi M, Tan Roberto, Tarkkanen Ahti, Teng K H, Tengroth Bjorn M, Tennant Jerald, Theodossiadis George, Thomas George, Thomson George, Thylerfors Bjorn, Tjan T T, Treloar Douglas, Trevor-Roper Patrick, Troutman Richard C, Uenoyama Kenshiro, Upali Mendis, Urrets-Zavalia A, Verdaguer Juan T, Vorosmarthy Daniel, Waltman Stephen, Wan Lee Seng, Wani Anil R, Watson Peter G, Weber M K, Wenas Harry, Wessing Achim, Wilson John, Woo C P Victor, Worst Jan G F, Yamanaka Akio, Yavuz Ulgar, Yeoh Lam Soon, Ron, Zagorski Zbigniew

\section{Supporters and volunteers from the World Cataract Surgeons Society}

Africa Ntim-Amponsah C T, Sharma M K, Australia Cowle Jeffre B, Gillies W E, Graham Peter, Lam Godfrey K Y, Mullins Michael, Nandor Jaroos, Tamblyn D M, Austria Nepp Johannes, Walter Richard, Waltersdorfer R, Widder Wolfgang, Bahrain Ghosh Tarak, Bangladesh Aftab-VZ-Zaman QM, Ahmed Shafuddin, Hug Faziul, Hyder Rashid, Islam Mahbubul, Islam Maksudul, Islam Shariful, Khalea Zafar, Khan Afzal, Kutubi Osman Shaeed, Mahubar Rahman, Matin M A, Pintu Saifuddin A, Rahman Mizanur, Shahid Hassan, Tariq Saifuddin M, Uddin Shahab, Barbados Kazi Mohammed A, Belgium Chris Deman, Koller Peeters, Brazil Avler Paulo Edvardo, C da Cunha Marcos, Marcia Tartarella, Tozatti Marra SV, Zinsly Bolivavl, Brunei Darusalam Joshi Nayan, Bulgaria Gougutchkova, Koev, Vassileva Petja, Canada Abdeen Az, Armogan Narendra, Beiko G H H, BragaMele R, Chan Jean, Cheung John, De Souza S, Farrell Margaret, Goel Jai Nl, Haley Gladys, Hunter W S, Hussein Naushad, Jans Ronald G, Johnson Ernest A, Kirker G E Mervyn, Koz Katherine Deanna, Lee Dickson, Marrae W G, Morgan Rod A, Murphy Madeleine Hay, Pierre M F Jean, Rabinovitch T, Ro Athanasius, Rootman David, Shin John, Styvie Quellet, Taylor Garth A, Thomas Rogers, Townsend Anthony, Weinstock S Joseph, Zaharia Marian A, Croatia Fett Mijo, Grgic Roza, Robert S, Cuba Agviar Lazgro Perez, Ferrer Olivia M, Torres Marcelino Rio, Czech Republic Baartos Dusan, Pasta Jiri, Pavel Rozsival, Sarka Pitrova, Vladinir Beran, Denmark Niecsen Per J, Egypt Galen Hesham, Hanna Lucy Sharfik, Hussein Ali Hussein, Sewelam Ashnaf, Finland Leikola Johannes, France Claude Benhamou, Hostyn Partrick, Pierre Garraud Wic, Hongkong Cheng P M George, Chor Fatt Tse, Goldschmidt Ernst, Hui Siu-Ping, Kwok Kay Tse, Lam S C Dennis, Liu King Yu, Po Chun Chan Pauline, Woo Chai Fong, Hungary A'Gnes Szabo 1, Degi Rosa, Futo Gabor, Grosz Andor, Gyetvai Tama’s, Hidasi Vanda, Horoozi Zoltan, Lajes Kolozsvari, Nemeth Janos, Papp Andrea, Pelle Susanne, India Agarwal Agar, Agarwal Athiya, Agarwal N K, Agarwal Sunita, Agarwala Nisheeta, Ahkaury R K, Amitava Abadan Kman, Azad Rajvardhan, Bansal Aashish K, Bharti S, Bhlishan Bharat, Chakravarty Somnath, Desai N C, Dubay Harsh, Dwevedi P C, Jilani F A, Khare M K, Kothari Kulin J, Mittal Om Parkash, Mulik Shivaraj, Namperumalsamy, Narendra Patel, Natarajan, Natchiar G, Nath Dharmendra, Pasricha J K, Prasad Shyam Sunder, Reddy K Madhukar, Saini J S, Sainil J S, Sen Sanjay, Sethi Arun, Shukla Bhushan, Sohanraj, Somdhi Pankat, Thomas Iayan, Thomas Jayan, Treman Vijay Part Ap, Uboweja Anil Kumar, Venkataswamy G, Yadav H N, Indonesia Budihardjo, Hoetarjo Nannerl, Iskandar Abizak, Mahdi Hariyah M, Putu Budhiastra, Salamun DSM, Simarmata M, Soewono Wisnujono, Susila N K Niti, Sutrisno Bamby, Iran Aminolah Nikeghbalz, Mohammadzadeh GH, Moheb Davood, Nikeghbali A, Samiee Sossein, Shams Mehdi, Israel Spierer Abraham, Italy Acfres Reibaldi, Carlevale Carlo, Enrico Gallenga P, Gianluca Scuderi, Marcello Santocono, Massiho Filippello, Picardo Vittorio, 
Tognon Sofia, Turrini Barbara, Uva Maurizio G, Japan Kinno Yasuhiro, Sato Tsuyoshi, Shiwa Toshihiko, Jeddah Zia U Khan, Jordan Fathi a Adas, Nafiz Sirhan, Yahya D Othman A, Kenya Reshef Danie, Korea Wansoo Kim, Kuwait Subhash Goswamy, Latvia Mairo Oibede, Jgor Solonivatim, Lebanon Chikhani Walid, Maine Metz Gerald A, Malawi Chipambo M C, Malaysia Au Mun Kit, Chin Pik Kee, Ching Wing Seng, Choo May May, Choong Yean Yaw, Dahalan Alias, Kang Dennis, Kiew Chit Choa, Krishnan Hari, Majid Mohd Wadzik,

Majurnder Ajit, Musadiq Mohammed, Muthusamy P, Pall Singh, Pillai Balaravi, Ramani Veera Dato, Ramasamy Rajagordi, Reddy T N Khrishna, Saad Ahmad Mt, Selvi, Shriwas S R, Sidhu Manjit S, Singh Sarbjit, Sivagnanam, Sivaraj Rajamalika, Subrayan Visvaraja, Tan Khian Khvon, Tan Niap Ming, Vijaya Ravindran, Yeo K C Robert, Zulkifli Abdul Ghani, Mexico Gomex Jose Antonio, Medina Hector, Olhovich Irene, Vallejo Miguel A, Morroco Abdelovahed Amran, Myanmar Aung Mya, Aung Than, Nyunt Kan, Norway Dag Riiso, Herdis Garborg,

Oman Wasti S Arshad Ali, Zutshi Rajiv, Pakistan Ahmad Rashid, Farooq Muhammad Umar, Islam Ziaul, Jacob Salim Azhar, Khan M Aman, Mohammad Zia, S Saif-ur Rahman, Yaqin Mohammad, Zafar Iqbal, Papua New Guinea Verma Nitin, Philippines Agulto Manuel, Ancelas de Leon, Ang Sammy, Arribas Irwin C, Balderas Joanne J, Crongruillo Yasmymo, Cruz Paul D, Damingo Perfecto S Jr, Gahol Luisito, Henson Radul, Henson Ruben, Lim Bon Siong, Ruben, Malabag-Valdez Alma, Martinex A B, Mendola Banara Ann D, Milante Rollo, Ngm Michael S, Rivera Mary Katherine, Romero B D, Santog Marcelo F, Singson Roseny Mae C, Sison Michael, Tan Timothy, Yang Richard S, Poland Dorota Wygledowska, Gierek-Kazicka Stanislawa, Gierek-Lapinska Ariadna, Mrvkwa Ewa, Portugal Luis Perira, PR China Ding Ke Xi, Gu Xun Qing, Huang Pei Gang, Jiang You Qin, Li Shaozhen, Tang Shu Chen, Wang Qin Mei, Yanshen Hao, Zhao Kanxing, Rep of Panama Barraza res Caballero, Russia Khvedeliani David J, Saudi Arabia Abdul Moiz Shams, Ahmed Tawfik Ali, Osama Badeeb, Sialkot Amin Ullah Baig, Muhammad Azhar Qazi, Singapore Ang Chong Lye, Au Eong Kah Guan, Chan Tat Keong, Chan Wing Kwong, Chee Caroline, Chua Ee Chek, Currie Chiang, Goh Jon, Heng Lee Kwang, Ho Ke Wai Tony, Jap Aliza, Koh Adrain, Koh Lian Buck, Lim Kuang Hui, Lim Li, Low Cze Hong, Low Siew Ngim, Lynn Yeo, Oen Francis, Raymond Phua, Sangeeta Taneja, Siow Ka Lin, Tseng Peter, Wang Lee Yuen, Yap Soo Keong, Yeo Kim Teck, Slovak Republic Strmer Peter, Slovenia Dusan Pusnik, Logar Primoz, Trantisew Vesely, Spain Enrique Aleman, Lopez-Ramos Pedro, Temprando Jose, Sri Lanka Reggie Simon, Sweden Jakobssan Gunner, Switzerland Flammer Josef, Syria Habal, Samir G Antaki, Taiwan, ROC Hui Chun Tai, Ray Fong Lee, Thailand Chaiwai Teekhasaenee, Chanchai Sethasuovan, Juthathip Hiri-O-Tappa, Noppadol Khangrang, Prin Rojanapongpun, The Netherlands G Van Rij, Jan G F Worst, Turkey Ahmed Igagasioglu, Ayfer Ergim, D Sezin Karadede, Erol Turacli, Halik Talu, Kadir Eltutar, Mahmut Kaskaloglu, Papilla Medical Pte Ltd, Suleyman Kaynak, UK A While, Bertil Damato, D Denni Fiberesima, Dutton Gordon N, I H Makar, John Thomsit, Mackintosh Graeme, Mcleod Kyle Peter, Murray Stephen, P Rosen, Pitts John, S Sandramouli, Watson P G, Wu John, Ukraine Bobrova N F, Boitchuk Irina M, USA Adrain G Ten Cate, Alexidze Alexander, Booth Frances, Braig Rasso, Brown Ballie R, Brown Harry S, Christy Norval E, Cohen Kenneth L, Feldgoise Louis T, Fryczkowski Andy, Goins Gary D, Gonzalez-Sirit Rafael, H S Ivang, K Myint, Kollaritis Carol R, Linder Barry J, Lye Sum 'Tan Linder, O'Day David G, Park John H, Preecs Gordon R, Ren Qiushi, Santiago Antonio B Sibayan, Shah Harshad, Sheridan Selma J, Shofner Steewart, Simjee Aisha, Stephen Bernie, Venezuela B Milgrom, Iris Raquel, Bermuzal Ahaya, Rivds Armando, West Indies Hosein Robin

This book is also dedicated to the hundreds of colleagues, co-workers and leaders of organisations dedicated to the prevention of blindness. Supporters from the International Council of Ophthalmology (ICO), Asia-Pacific Academy of Ophthalmology (APAO), International Intraocular Implant Club (IIIC), AsiaPacific Intraocular Implant Association (APIIA). 
This page is intentionally left blank 


\title{
Foreword
}

\author{
When, as a result of the lack of international \\ human organisation, human misery and socio- \\ economic agony of millions of blind cataract \\ victims continue to increase in the poor areas \\ of our world, at a time when spectacular medical \\ advance can restore normal vision at low cost \\ to all the blind cataract patients, I will continue \\ to press for action, for change.
}

Professor Arthur Lim 


\section{Acknowledgment}

I am particularly indebted to my wife, Mrs Arthur Lim, for her scrupulous editing and for joining me in my vision against cataract blindness. I would also like to thank Dr Kwa Soon Bee, the Permanent Secretary (Health) and Director of Medical Services, Ministry of Health, for his unwavering support of my endeavours.

Dr Ang Beng Chong, my able and dedicated partner and his wife, Dr Su Hong Hai, and the nursing staff led by Sister Peck Chye Fong for supporting my efforts so strongly, at a time when the implant was uncertain. The late Mr Chan Seng Poh and his daughter, Ms Chan Poh Choo for their generous donations, and Ms Charity Wai and Ms Chiam Soo Lee for their continued efforts in the communication and administration of the activities.

The coordination and fluency of the text is made possible through the efforts of Ms Lim Sook Cheng, the scientific editor of World Scientific Publishing Co Pte Ltd. My secretarial staff especially Tan Sok Huang and Jasmine Bu, have been extremely supportive throughout the duration of my writing. To them I express my gratitude.

I have WordMaker Design Pte Ltd to thank for the design and visual layout support, especially Mr KC Chew and Ms Raquel Rivera for their expertise in this area. 


\section{Contents}

Foreword

Chapter 1 Introduction

Chapter 2 Impact of Technology on Mass Blindness in Asia

Chapter 3 Increasing Importance of Eye Surgeons In Mass Cataract

Blindness - A Megatrend

Chapter 4 Tianjin Centre - Training of Surgeons

Chapter 5 Quality Assurance in Cataract Surgery

Chapter 6 Towards Perfect Outcome

Chapter 7 Low Cost Implant Techniques

Chapter 8 Quality Cataract Surgery in Asia in the Year 2000

Chapter 9 Interview With Professor Arthur Lim On Mass

Cataract Blindness

Afterword 